\title{
Correction to: Utilization of a Wheat55K SNP array-derived high-density genetic map for high-resolution mapping of quantitative trait loci for important kernel-related traits in common wheat
}

\author{
Tianheng Ren ${ }^{1}$ (1) Tao Fan ${ }^{1} \cdot$ Shulin Chen ${ }^{1,2} \cdot$ Chunsheng $\mathrm{Li}^{1,2} \cdot$ Yongyan $\mathrm{Chen}^{1,2} \cdot \mathrm{Xia} \mathrm{Ou}^{1,2} \cdot \mathrm{Qing}^{1} \mathrm{Jiang}{ }^{1,2}$. \\ Zhenglong Ren ${ }^{1,2} \cdot$ Feiquan Tan $^{1,2} \cdot$ Peigao Luo ${ }^{1,2} \cdot$ Chen Chen · Zhi Li ${ }^{1,2}$
}

Published online: 9 February 2021

๑) Springer-Verlag GmbH Germany, part of Springer Nature 2021

\section{Correction to: Theoretical and Applied Genetics https://doi.org/10.1007/s00122-020-03732-8}

Unfortunately, the legends of Figures 1 and 2 were missed out in the original publication. The legends are given below.

Fig. 1 Chromosomal locations of the stable QTL associated with KWPS, SN, KDR, KW, KL, TKW and PH based on a 371 RIL population derived from a across between CN18 and T1208. The QTL clusters were marked clearly on the chromosomes.
The original article can be found online at https://doi.org/10.1007/ s00122-020-03732-8.

Tianheng Ren renth@sicau.edu.cn

$\triangle$ Zhi Li

lizhi@sicau.edu.cn

1 College of Agronomy, Sichuan Agricultural University, Wenjiang, Chengdu 611130, Sichuan, China

2 Provincial Key Laboratory for Plant Genetics and Breeding, WenjiangChengdu 611130, Sichuan, China
Fig. 2 Chromosomal locations of digenic epistatic QTL for $\mathrm{PH}, \mathrm{TKW}, \mathrm{KL}, \mathrm{KW}, \mathrm{KDR}, \mathrm{SN}$, and KWPS.

Publisher's Note Springer Nature remains neutral with regard to jurisdictional claims in published maps and institutional affiliations. 